\title{
The Use of Video Data in Reading Research
}

\author{
Brian Rowan, Bridget Maher, Mark White \\ School of Education and Institute for Social Research \\ University of Michigan
}

This paper discusses the uses of video data in research on reading as well as future directions for research in the area. In the paper, we view video as a medium for collecting, transmitting, and storing research data, just like photography, audio recording, live observation with field notes, artifact collection, in-person interviewing, questionnaires, and administrative records are media through which data are collected, transmitted, and stored. The purpose of this paper is to point the reader to some existing resources on the collection of video data and to discuss some trends in the use of video data in research on teaching and learning in the area of reading. Our central argument is that video recording technologies are evolving rapidly, providing particular affordances-and challenges-for the use of video data in qualitative and quantitative research on reading. We argue that some of these affordances might also help address the "gaps" in reading research identified in this Handbook, gaps that now exist with respect to what has been studied in reading research, gaps in the translation of research findings from the reading research to reading practice communities, and gaps in how results from reading research are implemented in schools and classrooms. As we discuss below, new affordances in video data collection, management, and data analysis have the potential to address these gaps by allowing for research on new topics in the field, by allowing for different ways to communicate research results to different communities of practice, and by providing different ways to guide practitioners as they implement findings from reading research in classrooms.

Resources Describing Video Data Collection and Use in Research

Several publications have described the growing (and varied) uses of video data in research on teaching and learning broadly. A review by Erickson (2011) discussed the emergence and early use of video data in qualitative and ethnographic research. Stigler and colleagues discussed the use of "video surveys" in comparative cross-national research on teaching (Jacobs, Kawanaka, and Stigler, 1999; Stigler, Gallimore, and Hiebert, 2000). An edited volume by Kane, Kerr, and Pianta (2014) included several chapters on methodological issues arising in video-based studies of teaching effectiveness and their bearing on the practical evaluation of teachers. Goldman and colleagues' (2014) booklength monograph described the uses of video data in learning sciences research, and an edited volume by Janik and Seidel (2014) described the use of video data in European research on classroom teaching and learning.

Overall, this published work points to some special affordances of video in comparison to other modes of data collection for research on teaching and learning generally and, by inference, for reading research. Like direct observation, video data can be used to record the rich detail of real-time events. Of course, the features of events actually captured by video data depends on how, and how often, video tools are deployed, but holding those important features of research constant, video records preserve the exact timing and sequencing of observed events with less editing and more contextual detail than 
photographs, audio recordings, field notes, transcriptions, interviews, or questionnaires. Moreover, unlike most other data collection tools, video data are used in both qualitative and quantitative research. In qualitative research, video preserves the immediate visual and auditory phenomena inherent in events, allowing researchers to revisit basic data as they develop and refine coding schemes and check on the internal validity of developing hypotheses about a case. In quantitative research, especially large-sample studies of teaching practice, the preservation and re-use of video data allows analysts to more easily (and less obtrusively) deploy multiple raters to code the same events, allowing for estimation and correction for rater error in data analyses. Video also facilitates simultaneous use of multiple, structured coding schemes to characterize the same teaching and learning events, helping validate score interpretations arising from observation instruments. Further, in both quantitative and qualitative research, setting participants (or others) can view video recordings of events, and participant insights can be used (alongside observer accounts of the data) to better understand event participants' motives, cognition, decision-making, or knowledge use during the events under analysis. Finally, video data can be clipped and processed in a variety of ways that allow researchers to communicate their results visually—both to researchers and to practitioners.

\section{Evolution of Video Technologies}

Although many studies in education have used video data, we expect the number of such studies to increase rapidly in the next decade, both as a result of the rapid advances in video recording technology evident in the research reviews just cited and because developments in video technology allow researchers to address research questions they could not use this technology to study previously. Much of this is due to a continuing miniaturization of video and audio recording devices, as well as continuing decreases in the costs of purchasing good video resolution, and the improved compatibility of video devices with other digital devices. Because of these trends, camera equipment has become easier to transport and set up. In many research settings, a trained camera operator is no longer required because respondents (or analysts) can easily position, turn on, and use video equipment according to research protocols. Multiple cameras can easily be deployed in classroom research and video subjects can wear pedants that allow cameras to follow them as they move around (Derry, 2007). Some camera systems even remove the need to make decisions about zooming and panning the camera during recording as they allow 360 degrees of panning and zooming upon video playback (see DIVER project; Pea, et al., 2004). Cameras can even be mounted on respondents' heads to capture what they see (BlikstadBalas \& Sorvik, 2015; Blikstad-Balas, 2017; see also Burris, 2017 for a broader discussion of 'Point-of-View' camera usage). Connections to other equipment (like tablets or laptops), along with growth in internet access and bandwidth, make moving video records from field site to laboratory easier. Moreover, video data files are now more easily processed and analyzed using commercially developed and widely available tools for clipping, annotating, coding, and organizing video data (Derry, 2007). Finally, the costs of storing large quantities of video data have decreased and should continue to do so in the future.

Such advances in video technology may allow for reading researchers to deploy cameras across multiple contexts more efficiently and to conduct multi-site research more affordably. This, in turn, could provide insights into how different classrooms, teachers, and schools implement reading curricula, research-based teaching strategies, or other 
approaches to supporting readers. Researchers would also have the ability to use video as a part of understanding individual readers within a single classroom through use of evolving video technologies. Indeed, it is only recently possible to position multiple cameras (or even individual student cameras) so as to simultaneously capture and then sync video records of classroom dynamics, reading approaches, and interactions. The affordances are thus many and varied for reading researchers, as the opportunities to develop a complex record of classroom and readers' processes is only a recent possibility.

\section{Video Data in Qualitative Reading Research}

Previous volumes of the Handbook of Reading Research have mentioned the use of video as an approach to data collection and analysis but have not had a chapter focused on these video research methods. However, our review of qualitative (and mixed methods) studies in the field of reading research over the last 10 years found that video data have been used to investigate a range of substantive topics in the field. We found studies using video data that: documented reading and writing processes among young people using multiple and varied texts (e.g., Cho, Woodward, \& Li, 2018; Goldman, et al., 2012; Ivey \& Johnston, 2013); examined instructional and pedagogical practices in the service of reading and literacy (e.g., Aukerman \& Schuldt, 2016; Magnusson, Roe, \& Blikstad-Balas, 2018); analyzed language exchanges, discourse, and other detailed and complex interactions in classrooms (e.g., Moore \& Schleppegrell, 2014); and investigated the implementation of literacy interventions (e.g., Amendum, Bratsch-Hines, \& Vernon-Feagans, 2018; Levine, 2014). As in prior research, video data in the studies we reviewed were used to document phenomena in rich detail and to attend to patterns and meaning within and across video records. Video data were also used alongside other data sources to triangulate findings.

Reading researchers have also used an extensive body of data analysis practices with video data, but several fundamental issues remain unresolved. One of the most vexing issues arises from qualitative researchers' interest in coupling video with other data sources to arrive at a "thick description" of events and cultures (Geertz, 1973). As video data become more available to qualitative reading researchers, questions about the validity and reliability of thick descriptions drawn from video data remain. Just how much video data is needed to arrive at "thick" description? Can someone not present in the community at the time of events, or not fully a member of the community, truly understand, analyze, and interpret the sociocultural (or even temporal) context of phenomena under study using video? What specific aspects of sociocultural and temporal context, community context, or social interactions can video capture well and which does it ignore? How might video records be treated similarly or differently from the interpretation and analysis of field notes, photographs, and other media? How might classroom video document (some) aspects of reading, literacy, and interaction, while at the same time failing to document other aspects, and what choices would a researcher make in light of such limitations? Answers any of the questions raised here depend, of course, on a reading researcher's specific theoretical perspective, research topic, and methods of video data analysis. In our view, what is needed moving forward is more explicit discussion of these issues within specific reading research communities. It would also constitute growing evidence of maturity in the use of video data in the field.

Video Data in Quantitative Research on Reading 
Over the past decade, video data have also come into wide use in quantitative research on teaching, especially research using structured observation instruments to examine classroom instruction. The miniaturization of video equipment, and the easy setup and operation of such equipment by teachers, have made live observation of teaching less necessary and led to innovative uses of camera (and audio) equipment. The positioning of equipment is quite variable in this research, sometimes involving use of panoramic cameras, sometimes synchronization of cameras at the front and back of a classroom, sometimes the distribution of cameras to capture group work, and sometimes the mounting of cameras on students to capture the texts they are interacting with as they read or work on computers. The videos from all cameras can be synchronized, and as a result, many concerns about what can (or cannot) be captured in video studies of classrooms has been addressed (Derry, 2007). Still, the positioning of cameras depends in large part on the research questions being addressed, and we expect camera-positioning strategies to change in the coming years. What we hope evolves is some consensus about best practices in deployment of camera and video recording equipment in research on the teaching and learning of reading. However, best practices for video capture should be based on rigorous comparisons of alternatives. Unfortunately, comparative studies of video technologies and their effects on basic research processes such as measurement and statistical analysis is largely missing in current research. Thus, herein lies an additional opportunity for reading researchers to make progress in the field.

Beyond data collection, a major benefit of video data collection in quantitative research on classroom instruction is the increased ability to store video data for use in training and on-going certification. In research, viewing videos is now a central part of classroom observer training and calibration, with groups of observers frequently scoring common videos previously coded to a "gold standard" by experts. In heavily managed research projects, the scoring of videos is also sometimes monitored in real time, and coders who make large errors are pulled from scoring until they are re-certified (Park, Chen, and Holtzman, 2014). Video data collection further allows for easier (and less obtrusive) estimation of rater error in classroom observation studies than live observation because two or more raters can now score videos without those raters being present in the same classroom on the same day.

Collection of video data can also address another methodological problem in classroom research - the fact that both the quality of instruction and the ways observers score instructional quality often change systematically over time (e.g., Casabianca, et al., 2013). With live observation and live scoring, there is no way to disconfound these potentially correlated trends, and this makes it impossible to study changes over time in teaching practice using live observation (without large assumptions about time trends in rater error). With video scoring, however, videos for particular days can be distributed randomly to raters, making time of video recording and time of scoring uncorrelated.

Despite these affordances, some fundamental questions remain about the structured scoring of videos in reading research. For example, research suggests that video and live observations of the same classroom teaching sessions are scored differently (Casabianca, et al., 2013; Curby, et al., 2016), although the correlations between live and video scores for teachers are often quite high after adjusting for measurement error. Interestingly, items measuring features of instruction that involve social interaction tend to show the largest differences between live and video scoring, but this does not appear to be affected by issues 
related to audio or video quality (White, 2017). Beyond these few studies, however, little research has explored why scores might vary across live and video observations or whether such variation differs across particular observation systems. Holding constant the sampling features of live and video research design, it is possible that differences between live and video scoring are due to scoring protocols (e.g., raters can pause and revisit videos but not live events). Alternatively, differences could vary across specific camera systems used in a study or be sensitive to the observation instruments in use.

These last points are especially important because, with the rapid change in video technology, each new project seems to use a new camera setup, and yet, we are unaware of any studies comparing the impact on scoring of different camera systems. It would not be surprising, of course, to find that different camera systems, which create different opportunities to see the board, the teacher, student-to-student interactions, and what students are actually reading and doing during lessons have large impacts on observation scores or to find that differences vary across observation instruments. Thus, in reading research, where cumulative evidence from many studies is often used to evaluate the effectiveness of particular reading interventions and practices, it would be useful to give careful attention in reporting of study results to the equipment, observation instruments, and scoring procedures used in particular video studies. Such attention could help the field better understand any potential relationships between research results and video data collection/analysis procedures and might ultimately results in more agreement about best practices for video research within the field.

\section{Other Developments in the Use of Video in Research}

Other developments in the use of video in research are worth mentioning briefly, and we offer these as a way for imagining how to address various "gaps" in the field mentioned earlier. To begin, advances in software and technology for processing and streaming videos has made it easier to embed short clips of classroom practice within questionnaires. This has led to the embedding of video scenarios into surveys intended to measure teachers' knowledge, allowing teachers to respond to video representations of practice rather than text-based representations (Jamil, Sabol, Hamre, \& Pianta, 2015; Kersting, 2008). Video data are also being with other kinds of data as well. Mobile eyetracking technology, which overlays a teacher's gaze onto video data, is the most prominent example (see Beach \& McConnel, 2018 for a review of this research), but we expect other combinations to become common, such as the synchronization of video data with screen capture technology that can provide new viewpoints on how students work with technology-based reading interventions.

Technology is sure to develop in other interesting ways as well. One interesting path is the automatic processing and coding of video data. Commercially available software already exists to automatically code facial expressions and (animal) behavior from videos (e.g., www.noldus.com). While these applications currently require the object under study to be center-frame and facing the camera, technology is sure to develop to reduce this limitation. Further, there are attempts in sports broadcasting to develop technology that tracks objects (i.e. a soccer ball) and players in real-time from video data. Technology for automatic transcription of audio is also progressing quickly and may soon develop to the point that conversational turn-taking, wait time, and the timing of discourse events can be coded automatically. Last, the technology to create three-dimensional 
representations of what is captured on video has been available since at least the release of the X-Box 360 in 2005, which could expand the ways that video is used in research. Application of these new technologies to reading research remains to be explored.

\section{Archiving and Sharing Video Data}

A final set of issues in the use of video data in research are related to the increased demand by research sponsors, scholarly journals, and researchers to archive video data for re-use. The rationale and practices for archiving quantitative data from video studies are well established (see, e.g., Johnson, 2017). Issues associated with archiving and re-using qualitative data from video studies, however, are somewhat more controversial, even though practices for archiving qualitative data have been in place in fields outside of education for decades (see Irwin, 2013 for discussion). The archiving and re-use of raw video records, however, raise special concerns. At present, it is difficult to identify any venue other than local repositories to house video data from reading research, and the costs of storing large amounts of video data could slow the development of centralized institutional arrangements for video storage and re-use. A notable exception to this trend is the establishment of a video data enclave at the Inter-University Consortium for Political and Social Research, a virtual data enclave that houses video records from the Gates Foundation's Measures of Effective Teaching (MET) project (www.icpsr.umich.edu/icpsrweb/METLDB/). This enclave has been the site of many reuses of MET video data.

In addition to the complex problem of finding institutional support for video data archiving, there is currently no agreed upon template for the meta-data to be associated with video records (although standard templates make for a good start). Both the storage and meta-data problems make discovery and re-use of relevant video data in the field of reading research difficult.

Equally important are concerns about the privacy and confidentiality of the human subjects of video research, concerns that are making some school districts and many potential subjects of video research reluctant to participate in video studies. Much video research does not depend crucially on knowing the exact identities of participants, but shielding subjects from re-identification is obviously difficult in video work, raising questions about how much context data to associate with video data, how to share video data with other users, and how to use video exemplars of findings in published research (Derry, 2007). Technological solutions to some of these problems (e.g., altering video and audio in ways that obscure subjects' identities) are available, but the effects of such practices on data analysis and inferences from data are unknown. Compounding these problems is the wide cross-national variation in human subjects' protections, which makes comparative cross-national video research more difficult. In light of these problems, researchers planning to engage in video data collection need to think very carefully (and in advance of data collection) about these ethical and practical concerns. Particularly important is the crafting-well in advance-of consent forms for video research that clarify exactly how data will be used, by whom, and for how long.

The use of archived videos and video repositories could also serve as one approach to closing the translational gap in reading research. This gap exists when research findings in controlled, laboratory settings are not ultimately integrated into other research settings or into practice. As one example, My Teaching Partner, a coaching tool for classroom 
teachers, uses video illustrations of particular teaching domains from the observation tool CLASS-S (Gregory, et al., 2017). These videos are shared with novice teachers as they gain skills for beneficial teacher-student interactions; novices also share their own videos of attempts at these interactions as well as clips requesting feedback and support of a challenging issue in the classroom. Although My Teaching Partner did not particularly focus on reading research, it could model a possible direction of this research and use of video resources in the future.

Video libraries of reading instruction-videos within controlled research settings or videos of the translation of reading instruction to classroom environments-could serve as helpful training and illustration tools for practice and future teaching. Labeling and "tagging" video collections (or segments of videos) can provide helpful ways for teachers and instructors to search within archives for particular practices related to reading and teaching (see MET-X video repository within the Teaching and Learning Exploratory at the University of Michigan as one example). Video is often used in teacher education programs as assessment or reflection tools, but is typically not used beyond a single course or beyond a single teacher's training or portfolio. Establishing a video repository of exemplary practice or illustrations of specific practices may prove useful in demonstrating reading and literacy instruction, especially in ways that could establish common language and understandings around teaching and learning in reading. Teacher education programs could create examples of the development of teachers-in-training to show what is possible, even if not yet at exemplary practice, particularly around the area of reading instruction. Researchers and teachers alike could use such video resources to begin sharing practice and establishing shared language and vision about reading instruction and those beneficial practices to best serve students.

\section{An Example of Video Data in Research}

As a way of offering one example of the use of video data collection and analysis advancements, we describe a research project being conducted presently by the authors which uses video data collection, storage, and analysis tools as well as research practices described in this chapter. The study, which we call Teaching over Time (TOT), ${ }^{1}$ is a longitudinal, mixed-method study investigating the changes in teaching practice over the course of a ten-year period among a group of approximately 100 teachers of English Language Arts in grades 4 through 8 who earlier submitted video teaching records during the 2009-2011 school years as part of the well-known Measures of Effective Teaching (MET) study (MET Project, 2009). We followed up with a subset of 100 of these teachers during the 2017-2019 school years by collecting additional video records of teaching using a sampling plan similar to the one used in the MET study. We then coded all MET and TOT videos using the PLATO instrument for rating the quality of English Language Arts instruction (Grossman, Loeb, Cohen, \& Wyckoff, 2013) as well as a new scoring protocol that measured the extent to which teachers' lessons covered specific learning targets in the Common Core State Standards. As in many other studies, we also collected additional information about text use, approaches to reading, professional development experiences

\footnotetext{
${ }^{1}$ Teaching over Time is a part of a larger research effort entitled Under Construction: The Rise, Spread, and Consequences of the Common Core State Standards Initiative in the U.S. Education Sector. It is a Spencer Foundation and WT Grant Foundation endeavor across multiple universities and principal investigators.
} 
of teachers, the standards and objectives addressed in these teaching videos, and other details using both surveys and (in a subsample of teachers) interviews. Ultimately, we asked the question, how has teaching changed over the time interval of this study, and to what should we attribute these changes?

Our research effort offers one example of how a previous repository of videos has been resurrected for additional research efforts and research questions; such an opportunity would not be possible without the use of a storage repository with clear and useful metadata to find appropriate files. It also provides an example of the way classroom video might be deployed and collected across multiple sites, thus raising statistical power to make claims about what is happening in classrooms across multiple contexts. The study also shows how video camera equipment has evolved over the past ten years. In the MET study, a camera operator was present and a somewhat cumbersome panoramic camera was used for video recording. In TOT, by contrast, camera equipment was sent directly to teachers for setup and use, with technological support offered through remote communication. The TOT camera system also had multiple audio tracks to better record both student and teacher voices, and also allowed for the camera to automatically follow the teacher via infrared technology. Researchers on this study also conducted follow-up interviews with individual teachers as a part of the qualitative research component, allowing us to consider the influences, approaches, constraints, and affordances of different professional development opportunities, school contexts, curricular and instructional material, among others influences. The video data provided the research team several records of teaching practice from two time periods so as to provide a longitudinal record of approaches in classroom. In combination, we hope to use the video data, interviews, documents, and questionnaires to gain insight into the influences on English Language Arts teaching among TOT teachers. Ten years ago, such research would have been far more costly and difficult to conduct. It is only through increased miniaturization of camera and audio technologies, a decline in costs of these new technologies, and better ability to obtain low cost video storage and playback capacity that a study like Teaching over Time became feasible. We therefore anticipate additional developments in video research by other reading researchers and a growing use of video technologies and data in order to address existing gaps and remaining questions in the field.

\section{References}

Amendum, S., Bratsch-Hines, M., \& Vernon-Feagans, L. (2018). Investigating the efficacy of a web-based early reading and professional development intervention for young English learners. Reading Research Quarterly. 53(2), 155-174. https://doi.10.1002.rrq.188.

Aukerman, M. \& Schuldt, L.C. (2016). “The pictures can say more things": Change across time in young children's references to images and words during text discussion. Reading Research Quarterly. 51(3), 267-287. https://doi.10.1002.rrq.138. 
Beach, P., \& McConnel, J. (2018). Eye tracking methodology for studying teacher learning: a review of the research. International Journal of Research \& Method in Education, O(0), 1-17. https://doi.org/10.1080/1743727X.2018.1496415

Blikstad-Balas, M. (2017). Key challenges of using video when investigating social practices in education: contextualization, magnification, and representation. International Journal of Research \& Method in Education, 40(5), 511-523. https://doi.org/10.1080/1743727X.2016.1181162

Blikstad-Balas, M., \& Sørvik, G. O. (2015). Researching literacy in context: using video analysis to explore school literacies. Literacy, 49(3), 140-148. https://doi.org/10.1111/lit.12037

Burris, A. (2017). A Child's-Eye View: An Examination of Point-of-View Camera Use in Four Informal Education Settings. Visitor Studies, 20(2), 218-237. https://doi.org/10.1080/10645578.2017.1404352

Casabianca, J. M., McCaffrey, D. F., Gitomer, D. H., Bell, C. A., Hamre, B. K., \& Pianta, R. C. (2013). Effect of Observation Mode on Measures of Secondary Mathematics Teaching. Educational and Psychological Measurement, 73(5), 757-783. https://doi.org/10.1177/0013164413486987

Cho, B-Y., Woodward, L., \& Li, D. (2018). Epistemic processing when adolescents read online: A verbal protocol analysis of more and less successful online readers. Reading Research Quarterly. 53(2), 197-221. https://doi/10/1002/rrq.190.

Curby, T. W., Johnson, P., Mashburn, A. J., \& Carlis, L. (2016). Live Versus Video Observations: Comparing the Reliability and Validity of Two Methods of Assessing Classroom Quality. Journal of Psychoeducational Assessment, 34(8), 765-781. https://doi.org/10.1177/0734282915627115

Derry, S. J. (2007). Guidelines for Video Research in Education, 90.

Erickson, F. (2011). Uses of video in social research: a brief history. International Journal of Social Research Methodology, 14(3), 179-189.

Geertz, C. (1973). Thick description: Toward an interpretive theory of culture. In, The Interpretation of Cultures. New York: Basic Books.

Goldman, R., Pea, R., Barron, B., \& Derry, S. J. (Eds.). (2014). Video research in the learning sciences. Routledge.

Goldman, S., et al. (2012). Comprehending and learning from internet sources: Processing patterns of better and poorer learners. Reading Research Quarterly. 47(4), 356-381. https://doi.10.1002/rrq.027.

Gregory, A., Ruzek, E., Hafen, C.A., Mikami, A. Y., Allen, J. P. \& Pianta, R. C. (2017) My Teaching Partner-Secondary: A Video-Based Coaching Model, Theory Into Practice, 56(1), 38-45, DOI: 10.1080/00405841.2016.1260402

Grossman, P., Loeb, S., Cohen, J., \& Wyckoff, J. (2013). Measure for measure: The relationship between measures of instructional practice in middle school English language arts and teachers' value-added scores. American Journal of Education, 119(3), 445-470.

Irwin, S. (2013). Qualitative secondary data analysis: Ethics, epistemology and context. Progress in Development Studies, 13(4), 295-306.

Ivey, G. \& Johnston, P. H. (2013). Engagement with young adult literature: Outcomes and processes. Reading Research Quarterly. 48(3), 255-275. https://doi.10.1002/rrq.46. 
Jacobs, J. K., Kawanaka, T., \& Stigler, J. W. (1999). Integrating qualitative and quantitative approaches to the analysis of video data on classroom teaching. International Journal of Educational Research, 31(8), 717-724.

Jamil, F. M., Sabol, T. J., Hamre, B. K., \& Pianta, R. C. (2015). Assessing Teachers' Skills in Detecting and Identifying Effective Interactions in the Classroom: Theory and Measurement. The Elementary School Journal, 115(3), 407-432. https://doi.org/10.1086/680353

Johnston, L. R. (2017). Curating Research Data Volume One: Practical Strategies for Your Digital Repository. Association of College and Research Libraries.

Kane, T., Kerr, K., \& Pianta, R. (2014). Designing teacher evaluation systems: New guidance from the measures of effective teaching project. John Wiley \& Sons

Kersting, N. (2008). Using Video Clips of Mathematics Classroom Instruction as Item Prompts to Measure Teachers' Knowledge of Teaching Mathematics. Educational and Psychological Measurement, 68(5), 845-861. https://doi.org/10.1177/0013164407313369

Levine, S. (2014). Making interpretation visible with an affect-based strategy. Reading Research Quarterly. 49(3), 283-303. https://doi:10.10021.rrq.71.

Magnusson, C. G., Roe, A., Blikstad-Balas, M. (2018). To what extent and how are reading comprehension strategies part of language arts instruction? A study of lower secondary classrooms. Reading Research Quarterly. 0(0), 1-26. https://doi.org/10.1002/rrq.231.

MET Project. (2009). Learning about Teaching: Initial Findings from the Measure of Effective Teaching Project. Bill \& Melinda Gates Foundation. Seattle, WA.

Moore, J. \& Schleppegrell, M. (2014). Using a functional linguistic metalanguage to support academic language development in the English Language Arts. Linguistics and Education. 26(1), 92-105. https://doi.org/10.1016/j.linged.2014.01.002.

Pea, R. D., Mills, M., Rosen, J., Dauber, K., Effelsberg, W., \& Hoffert, E. (2003). The Diver Project: Interactive Digital Video Repurposing. IEEE MultiMedia, 11(1), 54-61. https://doi.org/10.1109/MMUL.2004.1261108

Stigler, J. W., Gallimore, R., \& Hiebert, J. (2000). Using video surveys to compare classrooms and teaching across cultures: Examples and lessons from the TIMSS video studies. Educational Psychologist, 35(2), 87-100.

Tomáš, J., \& Seidel, T. (Eds.). (2013). The Power of Video Studies in Investigating Teaching and Learning in the Classroom. BoD-Books on Demand

White, M. (2017). Generalizability of Scores from Classroom Observation Instruments. University of Michigan, Ann Arbor, MI. http://hdl.handle.net/2027.42/138742. 